$\S=-1$

\title{
Study of Performance of Real Time Streaming Protocol (RTSP) in Learning Systems
}

\author{
Yan Watequlis Syaifudin ${ }^{1 *}$, Imam Fahrur Rozi ${ }^{2}$, Rudy Ariyanto $^{3}$, Erfan Rohadi ${ }^{4}$, Supriatna Adhisuwignjo $^{5}$ \\ 1,2,3,4 Information Technology Dept., State Polytechnic of Malang \\ ${ }^{5}$ Electrical Engineering Dept., State Polytechnic of Malang \\ *Corresponding author E-mail: qulis@ polinema.ac.id
}

\begin{abstract}
Education is a very important aspect to develop the country to become more advanced. However, the constraints such as lack of education budgets have restricted many schools to invest in learning support equipment. One of the technologies in multimedia that can be used to build a learning system is media streaming that use a standard of implemented protocol is Real Time Streaming Protocol (RTSP). It can be used to build media streaming based learning model. This research will explain about the performance of RTSP if implemented in a classroom learning with capacity up to 50 hosts. Measurement is based on the performance aspect of the streaming process, including latency time, streaming lag, and CPU utilization. Parameters used to compare performance are media type, media format, media size, media quality, and number of clients. The combination of each parameter will produce different value of performance aspects. As a case study, foreign language subject is a learning activity that requires multimedia communication including video and audio, then this requirement can be solved with the development of media streaming based software using the RTSP. In the end it can be concluded about the feasibility of using RTSP to develop the learning system.
\end{abstract}

Keywords: performance, learning system, multimedia, LAN, RTSP, media streaming, audio, video, latency.

\section{Introduction}

Globalization requires every citizen to be able to master foreign language. Recently, the definition of educational technology has evolved and included the term "edutainment" which is point to mean the integration of "education" and "entertainment". The term edutainment promises fun and learning together [1]. Supported by the computer network technology and multimedia system that has increased the capacity, then the voice data and video can be processed and transmitted by computer. With the computer students do not just listen to the sound, but also be able to interact with the video system more attractive.

Learning Technology grew out of the practice of education and movement of audio-visual communication. Learning Technology is a combination of the three streams of mutual concern, namely the media in education, psychology of learning and approach in education system. One case study that can be modified using information technology is a model of learning a foreign language. With the use of multimedia systems, language learning can be more interactive. In English teaching, some teachers adopt multimedia teaching method without exception no matter what the teaching contents are. They haven't realized that multimedia teaching method is only an auxiliary one, and it is not the whole of the college English teaching [2]. The main feature of the language learning software is a feature of learning media. This feature makes it possible to display a variety of media, both text and multimedia media [3]. This feature makes it possible to display a variety of media, both text and multimedia format.

Multimedia combines five basic types of media into the learning environment: text, video, sound, graphics and animation, thus providing a powerful new tool for education [4]. Many types of media sources (audio and video) can be used for learning and vary greatly from aspects of media format, size, and quality. This aspect will greatly affect the performance of streaming if using RTSP. In addition, the number of online clients also affect the performance of the streaming process. Given multimedia-based learning using various types of media sources and the process is done massively (many students) it is necessary to analyze to measure the performance of streaming using RTSP.

\section{Technology in Streaming}

\subsection{Audio/Video Streaming}

The process of streaming media could be defined as the transfer of digital media (such as video, audio and data) that take place simultaneously with the application server that can be displayed in the client application in real time [5]. If the streaming process has been completed and the file has been displayed, the file is no longer available on the user's computer [3]. Streaming media is the process of sending data from the source (source) to the client is done in real time. This process runs continuously and does not require local storage media to store data [6]. The method as opposed to the method of download and play, meaning that the data is downloaded media ahead of the source and stored in a local storage media and then just run. So this process is not run continuously. Video and audio data capture results, will then be encoded or compressed with a particular format and saved in a file. The file is then deployed into the server to then be sent to the client. 
Multimedia (audio/video) streaming refers to the real-time transmission of stored multimedia contents. Streaming process itself is divided into two models, namely:

a) Live streaming, ie streaming process that takes source (video / audio) from input devices like webcam, microphone, camcoders, media player, and more. Video / audio is transmitted by broadcast or host to host from server device to audiences or viewers. The downside of this model is that there is time delay between the transmitter and the viewer. The use of this model is on media broadcasting, video chat, online meeting, and more.

b) On-demand streaming, which is a streaming process that stores media file on the server and streams it to each audience. With this model, viewers can watch the video / audio repeatedly at any time.

\subsection{Real Time Streaming Protocol (RTSP)}

RTSP (Real-time Streaming Protocol) [7] is an application-level protocol to control the transmission of data that has real-time constraints through network [8]. The RTSP is also a non-connectionoriented protocol that uses a session associated with an identifier [9]. RTSP is often used for streaming digital media, such as audio and video in real time. Beyond the sending requests from client to media server, RTSP allows the client to control real-time media steraming on the server as well [10]. The use of RTSP allows to view live video from direct sources (such as an encoder or camera) using a standard media player which compatible with the protocol, as show in Fig. 1. The definition of standard media player in this case is a media player that already implements RTSP standards that have been listed in RFC2326 [7]. Some examples of media players that have implemented the standard RTSP include Video LAN Client (VLC), Quicktime and many other media players that has implemented it. RTSP establish and control either one or several time-synchronized streams of a continuous media such as audio and video. It doesn't typically deliver the continuous live streams by itself, although the interleaving of the continuous media stream with the control stream is possible. In other words, RTSP acts as a "network remote control" for multimedia environment. The application of RTSP become wider nowadays, since most of multimedia mobile phones support it [11].

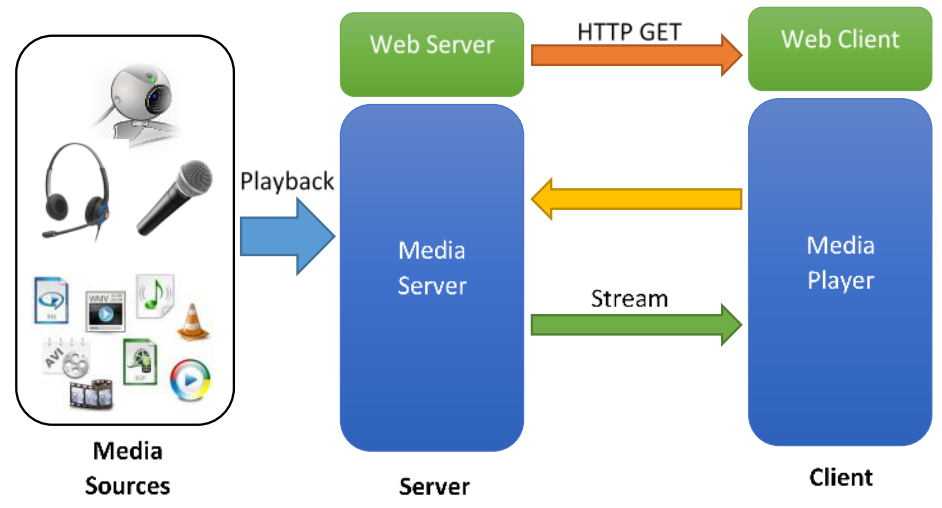

Fig. 1: Interaction between client and server using RTSP

\subsection{Technology in Language Teaching}

The use of computers and networks in language teaching has been widely proven positive impacts in language teaching. Language teaching in this case is the teaching of language skills that include listening, speaking, reading, writing, and teaching of the language component, namely grammar, vocabulary, and spelling. Therefore, network multimedia technology can greatly improve the students English comprehensive ability. The multimedia technology has the advantages of graphics, words and videos, and disseminates information in hypertext and hypermedia way [12]. With the use of computers and the Internet, learners can develop language skills broadest without being limited by time and space. In addition, learners can interact with fellow learners, the original addressees of English, and also with his English teacher [3]. However, subject to the limitations of traditional English grammar teaching model, universities less on teaching arrangements, limited time teachers teaching, blackboard and chalk this traditional teaching model also restricted the students' enthusiasm, this teaching method lead to students cramming it few can truly understand grammar knowledge, thus making the students did not fully grasp the English Language courses [13].

\section{Streaming Measurement}

To measure the performance of a streaming process, things to note are the factors that affect performance, the aspects that are used as performance benchmarks, and how to measure performance.

\subsection{Factors that Affect Performance}

Real time streaming in this discussion refers to the live streaming model, where the source of the media can come from live video / audio and live media player. Therefore, the performance factor that is a concern is the aspect of latency time, packet loss, and CPU utlization. The purpose of testing an RTSP streaming server is to obtain its interactive response ability and get its capability of dealing with concurrent media flows [8]. Some aspects that are predicted to affect the performance of the stream are:

a) Type of media source (live cam or media player)

b) Hardware specifications (processor, memory, LAN, and storage)

c) Network configuration

d) Number of clients online

e) Source file format: avi, mp4, mp3, wav, and others

f) Source file size

g) Media quality (bitrate for audio and frames per second for video)

\subsection{Measurement Methodology}

The purpose of performance testing of the streaming process in this discussion is to determine the feasibility of using RTSP on the learning process in the classroom using a multimedia source (audio / video). The class capacity standard will use up to 50 participants who will be streaming simultaneously. While from a media source type, the test will use a web cam device, a microfon, and various types of audio and video files. The sequence of activities for performing performance measurement is as follows:

a) Turn on all devices both server and client.

b) The server starts streaming and recorded initial time.

c) Client starts displaying broadcast results and recorded initial time.

d) Note the duration of the data loss.

e) The server does stop streaming.

\section{Testing Environment}

To test the performance of the streaming process using RTSP, we used a test environment at a standard computer laboratory that has been provided in many schools. The used software is a languagebased learning software that utilizes RTSP for streaming process. This software has been developed before and many use multimedia sources both audio and video for the learning process. 


\subsection{Software Architecture}

The features of the software consist of resource management, streaming media, group discussion and examination. Teachers have a role and a duty to control the activities of learning and teaching students in a language laboratory as the provision of learning materials, create quizzes, create a group for discussion, confirm their attendance to the students. In the streaming feature, there are two types of user levels, namely the control panel (Teacher) and Booth (Students). As shown in Fig. 2, a control panel is the teacher. Teachers have a role and a duty to control the teaching and learning activities of students in a language laboratory as the provision of learning materials.

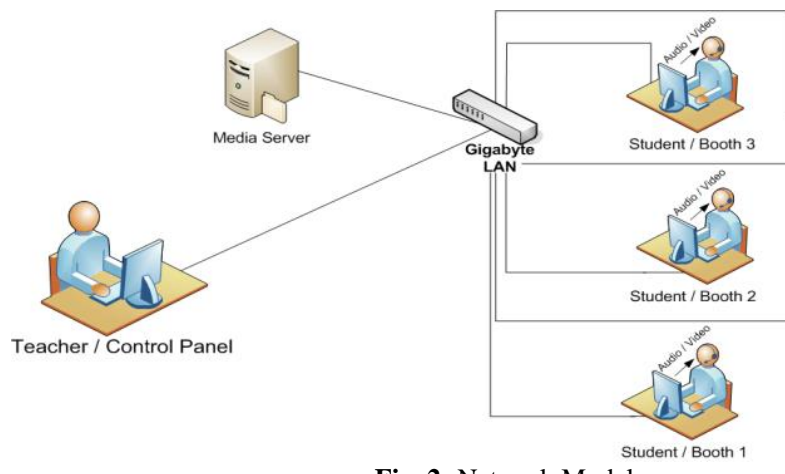

Fig. 2: Network Model

\subsection{Network Architecture}

While the definition of the booth is the student, where the student has a role to accept learning activities, each booth has one IP as shown in Fig. 2. Teaching and learning activities within the system uses the network via the LAN, where the LAN is a liaison between teachers and students, which means that when teachers will be conducting teaching in the language lab, the teacher will be entered into the system and take advantage of interactive learning media where learning materials such, will be distributed or shared to all students in a language laboratory through a LAN network. All students in the laboratory will be able to access the instructional media that contains a file of learning in the form of audio or video stored on the media server.

\subsection{Laboratory Specification}

In this testing process, we use the hardware in a school computer laboratory. The number of computers used is 31 units of computers divided into 1 unit for the moderator (teacher) and 30 units for the client (students), that shown in Fig. 3. The specifications of computer and network devices are as follows:

- Processor: Intel ${ }^{\circledR}$ Core $^{\mathrm{TM}}$ i3-2350M (Cache 3M, 2,30 $\mathrm{GHz}$,

- Memory: 4GB DDR3 PC-10600 ECC REG,

- Network interface: PCI LAN Card, Complies with the IEEE802.3u 100Base-TX and IEEE802.3ab 1000BaseT standards,

- Switch/Hub: 2 x Switch Hub 24-Port Gigabit.

- Others: Monitor, Speaker, Headset, and Webcam.

These are tools that have been used:

- Operating System: Microsoft Windows 10 Home Edition 64 bit,

- Web Browser: Mozilla Firefox,

- $\quad$ CPU and Bandwidth monitor: Task manager,

- Media Plugin: VLC

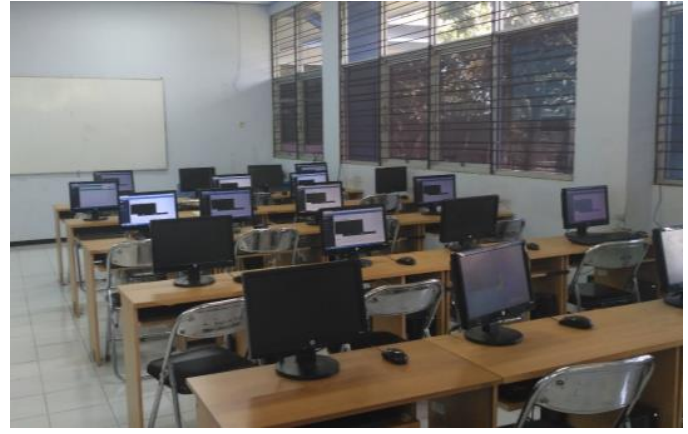

(a)

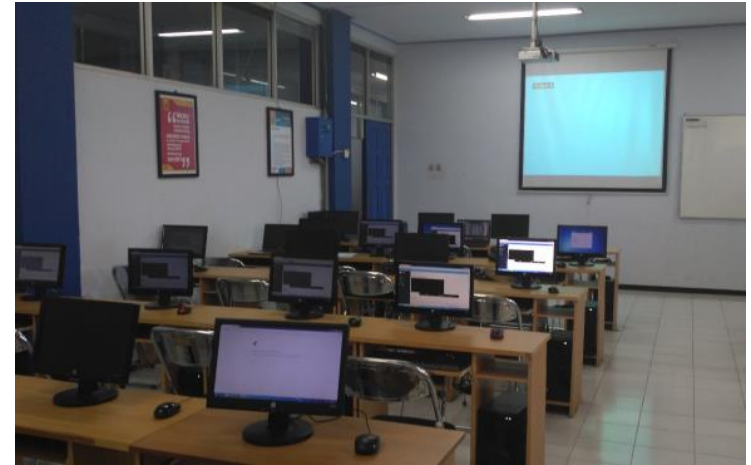

(b)

Fig. 3: Computer Laboratory

\section{Performance Testing}

\subsection{Testing Scenario}

Referring to section 3.1, the performance of the streaming process may be influenced by the type of media source, hardware specifications, network configuration, number of clients online, source file format, source file size, and media quality. Therefore, the test scenario to be performed will use variables from some of those aspects. The following is a breakdown of the variables to be used in the testing process, which is shown in

Table 1.

Table 1: Testing variables

\begin{tabular}{|c|c|c|c|}
\hline No. & Aspects & Definition & Variables \\
\hline 1 & $\begin{array}{l}\text { Type of me- } \\
\text { dia source }\end{array}$ & $\begin{array}{l}\text { Media sources are from record- } \\
\text { ed media files and live capture }\end{array}$ & file, live cam \\
\hline 2 & $\begin{array}{l}\text { Hardware } \\
\text { specifications }\end{array}$ & $\begin{array}{l}\text { The test will use the hardware } \\
\text { standard specification for the } \\
\text { computer lab described in sec- } \\
\text { tion } 4.3 \text {. }\end{array}$ & - \\
\hline 3 & $\begin{array}{l}\text { Network } \\
\text { configuration }\end{array}$ & $\begin{array}{l}\text { The test will use standard con- } \\
\text { figuration of LAN for the com- } \\
\text { puter lab described in section } \\
4.2 \text { and } 4.3 \text {. }\end{array}$ & - \\
\hline 4 & $\begin{array}{l}\text { Number of } \\
\text { clients online }\end{array}$ & $\begin{array}{l}\text { Number of clients will influ- } \\
\text { ence streaming performance. } \\
\text { We will do } 4 \text { steps to measure } \\
\text { it. }\end{array}$ & $\begin{array}{l}2,5,10, \text { and } \\
30 \text { clients }\end{array}$ \\
\hline 5 & $\begin{array}{l}\text { Source file } \\
\text { format }\end{array}$ & $\begin{array}{l}\text { Many format of video and } \\
\text { audio files that supported by } \\
\text { RTSP. We will use common } \\
\text { format that have been used to } \\
\text { record English learning. }\end{array}$ & $\begin{array}{l}\text { Audio: mp3 } \\
\text { and wav } \\
\text { Video: mp4 } \\
\text { and mpeg }\end{array}$ \\
\hline 6 & $\begin{array}{l}\text { Source file } \\
\text { size }\end{array}$ & $\begin{array}{l}\text { We will use } 3 \text { types of file size, } \\
\text { relatives to file format }\end{array}$ & $\begin{array}{l}\text { small, medi- } \\
\text { um, large }\end{array}$ \\
\hline 7 & Media quality & $\begin{array}{l}\text { We will use } 3 \text { types of media } \\
\text { quality. Audio format has bi- } \\
\text { trate. Video format has frame } \\
\text { per second (fps) and screen } \\
\text { size, but we only used fps be- } \\
\text { cause all of source media have }\end{array}$ & $\begin{array}{l}\text { small, medi- } \\
\text { um, large }\end{array}$ \\
\hline
\end{tabular}




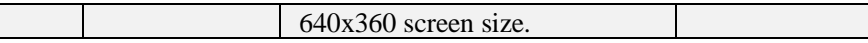

In accordance with the flow of software for language learning, the streaming process will start from the moderator computer that held by the teacher. From this computer, source media in the form of video, audio, and live cam will be broadcasted to all the online clients. All aspects of media resources (media type, file format, file size, and quality) will be tested for streaming process and recorded in performance measurement results.

\subsection{Performance Metrics}

To date, there are no fixed standard measurement metrics for the evaluation of streaming media service [8]. Based on the way performance measurement of client-server communications in a computer network and the performance of the audio / video streaming process, we define the performance metrics for streaming process on the computer network. Each performance metric will be generated from each test on each aspect variable that has been defined in section 5.1. Here are the metrics for measuring the performance of the streaming process:

a) Latency time (in seconds): time takes to transmit the packet data from the server to the client booth. In the streaming case, latency time is time that measured from moderator played a media source until clients responded and played the media streaming data. So, latency time is a total time from processing media sources, packaging stream data, transmit stream data, stream data delivered by client, until play stream data at client. The perfect latency time is 0 second, it is mean there is no delay between moderator plays the media and client presents the stream data.

b) Streaming lag (whether or not): video or audio on the client was not played continuously by clients.

c) $C P U$ utilization (in percent): with the increasing of streaming process, CPU unitilization in moderator and client will be increased.

Of course, software users for language learning want high streaming performance, consisting of low latency time, no lag, and low CPU usage. The experts of learning application determine the maximum tolerance for the latency or delay time is 5 seconds.

\subsection{Testing Results}

The test results are presented in graphical format showing latency time (in seconds) and CPU utilization (in percents). In accordance with the test scenario, the testing process is performed for each type of media source and other performance aspects (media quality and file size). On each test item, we measured streaming process performance metrics on 2, 5, 10, and 30 clients.

\section{a) Live streaming (from cam and microphone)}

Streaming process on live media is only done testing on webcam and microphone device with no lag. When testing performance of live video, we use both, while testing performance of live audio, we only use microphone. In this test scenario, no aspect of quality and file size was used. The live media process uses the standard h.264 format for streaming process. The latency time and CPU

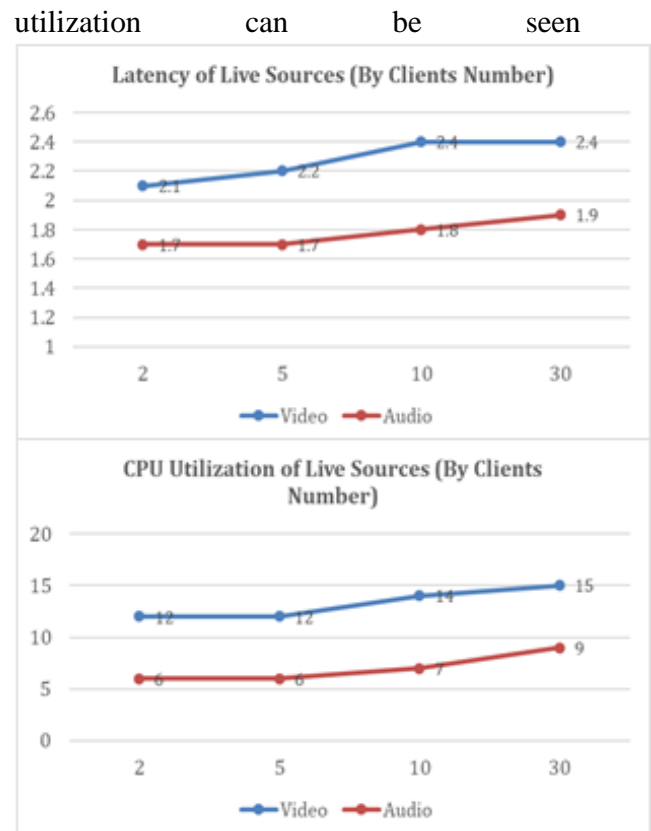

Fig. 4, as testing result.

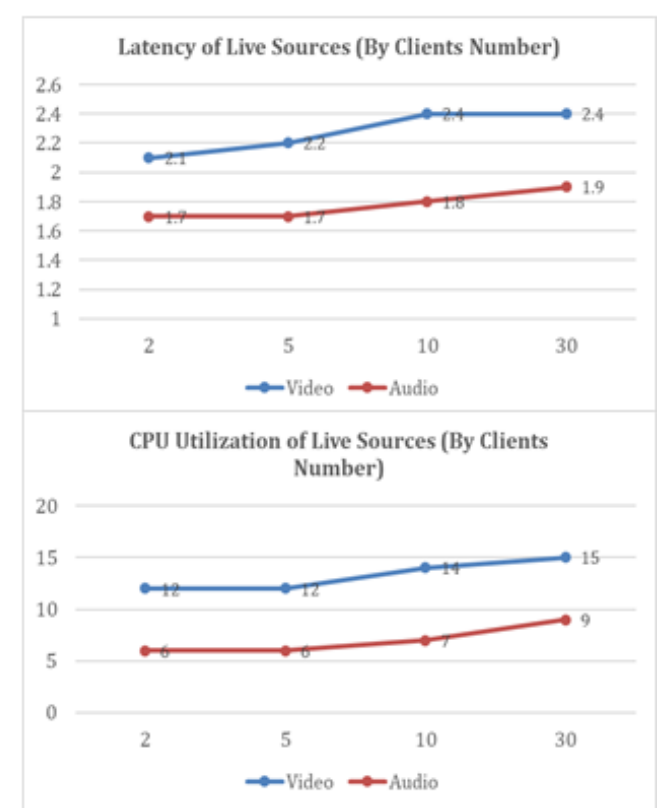

Fig. 4: Testing result of live media

b) Live streaming (from cam and microphone)

The audio source format is MP3 and WAV. These two formats are most widely used as language learning media. Bitrate (in bits) is an indicator of quality of an audio file. If an audio file has higher bitrate value, it has higher audio quality and can produce more detailed sound. We compared 3 bitrate values of each audio file 
format (mp3 and wav), and the values are 64, 128, and 256 bit. The latency time and CPU utilization can be seen in the Fig. 5, as testing result. All of clients have no lag when playing streaming media.

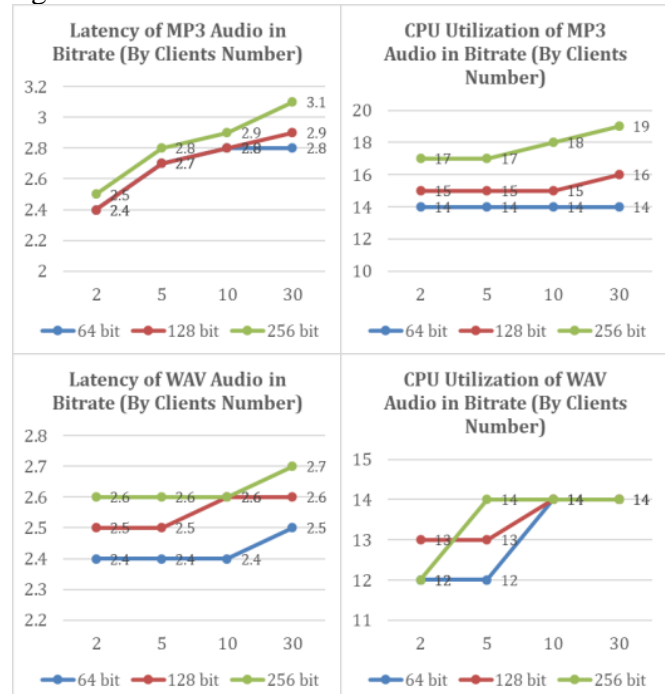

Fig. 5: Testing result from on-demand audio sources with different bitrate

c) On-demand video with quality aspects

The video source format is MP4 and MPEG. These two formats are most widely used as language learning video. Frame per second (in fps) is an indicator of quality of video file. If a video file has higher fps value, it has higher video quality and can produce smoother pictures movement. We compared 3 fps values of each video file format (MP4 and MPEG), and the values are 10, 25, and 30 bit. The latency time and CPU utilization can be seen in the

Fig. 6, as testing result. All of clients have no lag when playing streaming media.

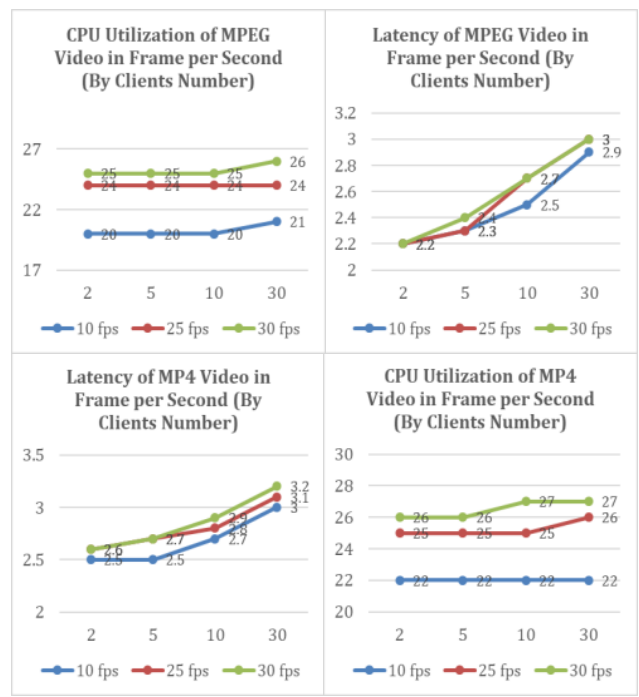

Fig. 6: Testing result from on-demand video sources with different FPS (frame per second)

\section{d) On-demand audio and video with filesize aspects}

All of audio source has bitrate 128 bit, as a standard value of bitrate. All of video source has quality $25 \mathrm{fps}$, as a standard value of fps. If an audio or video file has higher filesize value, it has longer time to play. We compared 3 filesize values of only MP3 audio file format, and the values are $\pm 1 \mathrm{MB}, \pm 4 \mathrm{MB}$, and $\pm 8 \mathrm{MB}$. We also compared 3 filesize values of only MP4 video file format, and the values are $\pm 10 \mathrm{MB}, \pm 50 \mathrm{MB}$, and $\pm 100 \mathrm{MB}$. The latency time and CPU utilization can be seen in the Fig. 7, as testing result. All of clients have no lag when playing streaming media.

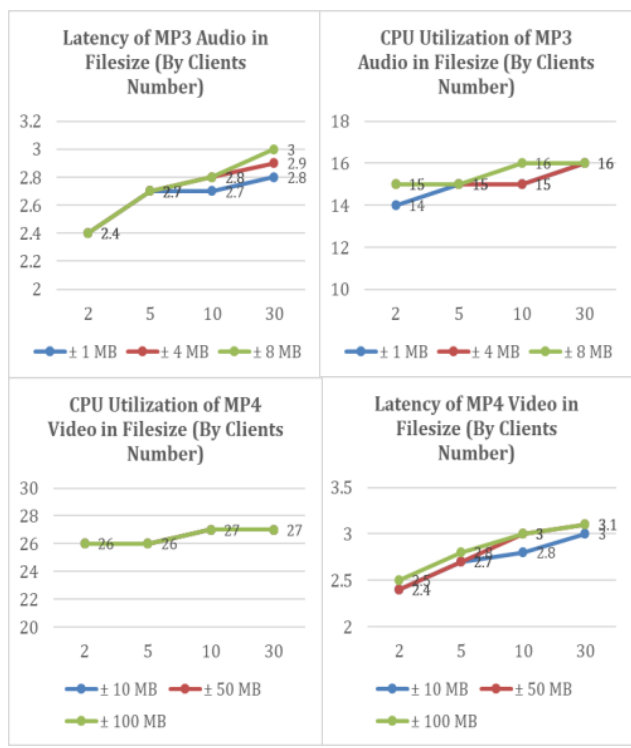

Fig. 7: Testing result from on-demand audio sources with different filesize

\section{Conclusion and Future Work}

In accordance with the test results, we can summarize the results are as follows:

a) The lowest latency time value is 1.7 seconds and the highest is 3.2 seconds.

b) No lag occurs when playing media on the client for all test sessions.

c) The lowest CPU Utilization value is $12 \%$ and the highest is $27 \%$

d) There is an increase in the latency time and CPU utilization values for each increase in the number of online clients, but stagnation occurs in certain events.

e) Video media produces higher latency time and CPU utilization values than audio media.

The latency time produced by all test sessions indicates that the RTSP protocol is still feasible for language learning using real time streaming in a computer lab (not more than 5 seconds). But the lowest latency value of 1.7 seconds is still quite high, so it needs improvements in terms of hardware and computer network configuration. The literature on interactive videoconferencing varies depending on the application areas [14]. This is understandable because a computer used for a communication node is not specifically used for communications only, but a computer also performs other processes. In addition, the process of streaming on language learning is done by broadcast, not point to point, so it will flood the computer network path when the streaming started. Results that corroborate the feasibility of using RTSP is that there is no lag when playing all media on all clients, so the information received by the user can be delivered entirely, nothing is truncated Similarly, the CPU utilization value is low enough for all test sessions, so it will not overload the processor even if a computer is used to run other software. Similarly, the CPU utilization value is low enough for all test sessions, so it will not overload the processor even if a computer is used to run other software. However, the use of video media, compressed media formats, and large media sizes will further increase the latency time so as to degrade the performance of the streaming process.

This test still covers only the basic needs of a software for learning using real time streaming, ie latency time, stream lag, and CPU utilization. In the future, we need to measure bandwidth usage and 
streaming media quality, if the software usage is wider in a WAN, internet, and cloud. The results of the test result suggest that the use of small audio media as well as in uncompressed format will further maximize the performance of real time streaming, especially if the number of clients is also small.

\section{Acknowledgement}

The authors would like to thank to Indonesian Directorate General of the Higher Education (DIKTI) and State Polytechnic of Malang who have supported this research.

\section{References}

[1] A. Verma, "Multimedia \& ICT in English Language Education," Journal of Technology for ELT, vol. 3, 2013.

[2] R. Susikaran, "The Use of Multimedia in English Language Teaching," Journal of Technology for ELT, vol. 2, no. 2, 2013.

[3] Y. W. Syaifudin, I. F. Rozi and A. N. Asri, "Web-Based Communication Model Between Teacher and Student in Foreign Language Software," Malaysian Construction Research Journal, vol. 2, no. Special Issue, pp. 231-240, 2017.

[4] S. Thamarana, "Use of Multimedia Technologies in English Language Learning: A Study," International Journal of English Language Teaching, vol. 4, no. 8, pp. 15-30, 2016.

[5] T. Hartsell and S. C.-Y. Yuen, "Video Streaming in Online Learning," AACE (Association for the Advancement of Computing in Education) Journal, vol. 14, no. 1, pp. 31-43, 2006.

[6] Y. W. Syaifudin, I. F. Rozi and A. N. Asri, "The Design of Foreign Language Teaching Software in School Computer Laboratory," Asia Pacific Journal of Contemporary Education and Communication Technology, vol. 2, no. 1, pp. 200-209, 2016.

[7] H. Schulzrinne, A. Rao and R. Lanphier, "RTSP: Real Time Streaming Protocol, IETF RFC 2326," Internet Engineering Task Force (IETF), 1998.

[8] Y. Liu, B. Du, S. Wang, H. Yang and X. Wang, "Design and Implementation of Performance Testing Utility for RTSP Streaming Media Server," in 1st International Conference on Pervasive Computing, Signal Processing and Applications, Harbin, China, 2010.

[9] I. Santos-González, A. Rivero-García, J. Molina-Gil and P. Caballero-Gil, "Implementation and Analysis of Real-Time Streaming Protocols," Sensors - Open Access Journal, vol. 7, no. 4, pp. 1-17, 2017.

[10] Z. Shraddha, J. Rahul, S. Rishikesh, A. Poorvi, "DREAM: A Data Streaming Application Using RTP/RTSP in a Local Network", International Journal of Engineering Science and Computing, vol. 6, issue no. 3, 2016

[11] K. Mohamed, A. Mahmoud, H. Chaafa, "Video Conference Android Platform by Your Mobile Phone", International Journal of Advanced Computer Science and Applications, vol. 6, no. 6, 2015

[12] Z. Hui-Min, "Study on The Constructivism Teaching Mode of College English Based on Multimedia Network Technology," in 2014 IEEE Workshop on Advanced Research and Technology in Industry Applications, Ottawa, Canada, 2014.

[13] Y. Wang, "Study of Application of Multimedia Technology in English Language Teaching," in 7th International Conference on Information Technology in Medicine and Education, Huangshan, China, 2015.

[14] S. Bhosale, V. Pottigar and V. Chavan, "A Review on Video Streaming in Education," International Journal of Computer Science and Information Technologies, vol. 6, no. 2, pp. 1088-1091, 2015. 